\title{
Investigation of Flow Characteristic on Ram Pump in Different of Pipe Junction Geometry
}

\author{
Stefan Mardikus ${ }^{1, *}$, Dwiseno Wihadi ${ }^{2}$, and ${ }^{3}$ Maryanto \\ ${ }^{1,2}$ Mechanical Engineering Department, Sanata Dharma University, Indonesia \\ ${ }^{3}$ Graduate Student, Mechanical Engineering, Sanata Dharma University, Indonesia
}

\begin{abstract}
Ram Pump is a mechanical device that uses phenomenon of difference pressure to distribute the water toward high state level. The advantages of ram pump are low cost, construction of reliable and without electrical energy. Kinetic and potential energy are main factor to flow the water in driver pipe. This research purposed to determine flow characteristic in different geometries of ram pump based on experiment data. Ram pump operated on different pressure of inlet area which was get from water level. To get the flow characteristic of rum pump, it was simulated using computational fluid dynamic. The result of simulation was found that junction type of $U$ shape appeared wake in driver pipe area; thus, this phenomenon indicated any turbulent flow because decreasing of pressure. In other junction pipe, wake phenomenon appeared in driver and delivery pipe area.
\end{abstract}

\section{Introduction}

Ram pump is a fluid flow device within pipe network as main tools that work to generate kinetic and potential energies. Through ram pump, water can be distributed without electrical energy. Several technologies still use source of energy from chemical energy of the fuel to produce potential energy and to lift the water [1]. Main principle of ram pump system based on level of water, power of falling water and design of ram pump. Design ram pump still need to be improved due to losses energy through characteristic of fluid flow that happened. Effect of pressure wave in ram pump body to influence force water. Design of ram pump can provoke to appear turbulent model and it will decrease output pressure. The construction of ram pump consists of ram pump body, impulse valve, pressure chamber, drive pipe and delivery pipe. Through the drive pipe, the water enter into the ram pump body, then water hit the impulse valve and the water flow to delivery pipe while the impulse valve closed. When the impulse valve is closed, it can increase the pressure water during a short period of time, meanwhile the impulse valve is opened, the water pressure is low [2].

During the water flowing in ram pump body, there is phenomenon of fluid flow like as turbulence that can give wake effect and this condition able to drop off the water pressure. In the present study, a numerical simulation that based on experimental data is investigated to know flow characteristic in ram pump body with varying of ram pump geometry models.
The schematic diagram of this experiment can be showed in figure 1. In this case, there are three types of ram pump geometry models like as types of Y-shape, Ushape and T-shape which have diameter of size is same.

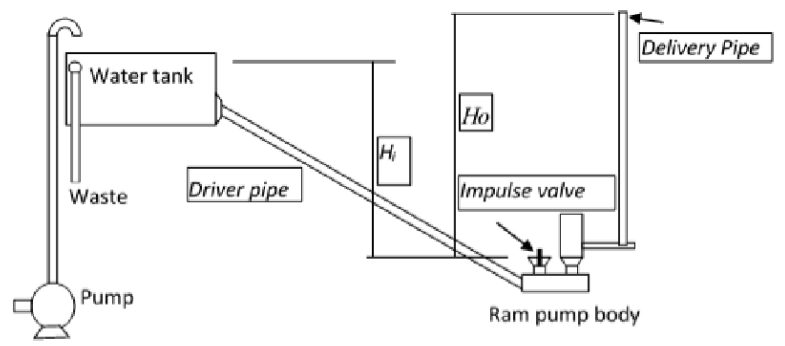

Fig. 1. The experimental schematic of ram pump system.

The computational mesh is generated by using ANSYS Meshing. Because of the complexity of the computational domain, tetrahedral mesh was used in this simulation. On the types of ram pump body, number of cell was different. Several parameter that would be investigated were distribution of pressure, distribution of velocity and turbulent intensity. Variation of dynamic pressure were given of 1.2 bars, 1.0 bars, 1.4 bars that was correlated with static pressure of 0.2 bars. The static and dynamic pressure was found by experiment data. Detail of experiment data that used can be provided by Table 1.

The computational domain consists of inlet region outlet region and wall fluid that be showed on Figure 2, Figure 3, Figure 4. The boundary conditions for all surfaces are described as follow:

\section{Methodology of Research}

\footnotetext{
Corresponding author: stefan@usd.ac.id
} 


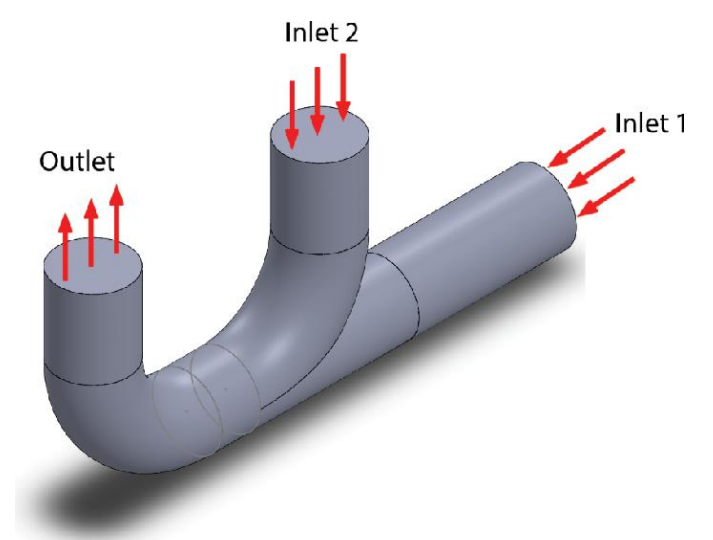

Fig. 2. Computational domain of U-shape model on ram pump bodies.

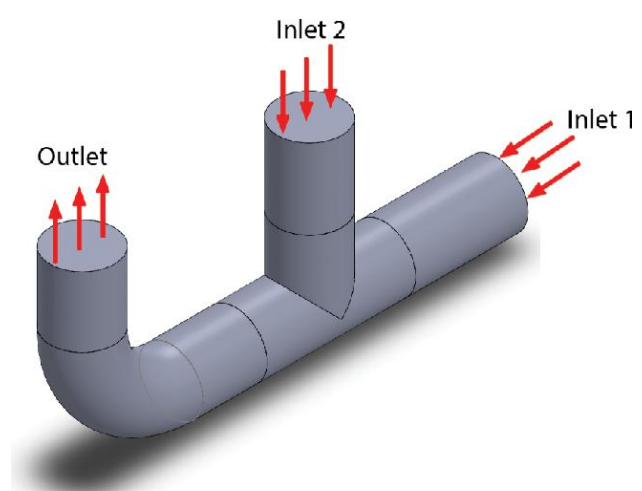

Fig. 3. Computational domain of T-shape model on ram pump bodies.

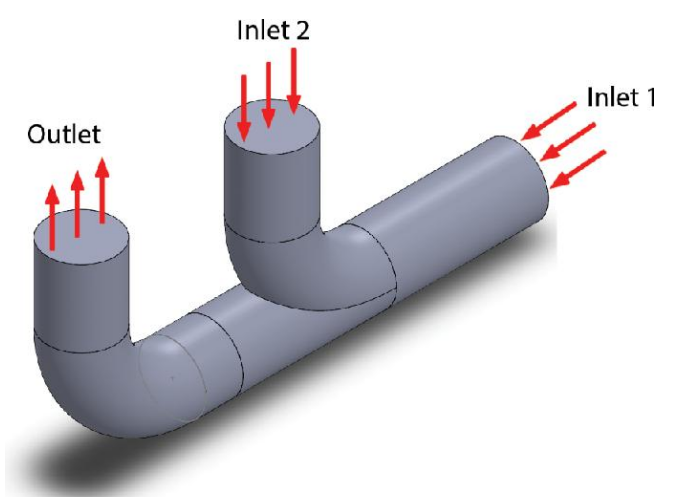

Fig. 4. Computational domain of Y-shape model on ram pump bodies.

In order to describe the mesh, it can be seen in Figure 5. The Navier-Stokes with boundary condition equation are finished by a computational fluid dynamics program (Fluent). Viscous model that be used on this solution was realizable K-epsilon model. For discretization of pressure-velocity coupling, it used type of phase coupled simple. The gradient of discretization type used least squares cell-based. Second order type was selected under discretization pressure and momentum. First order was selected for turbulent kinetic energy and dissipation rate.

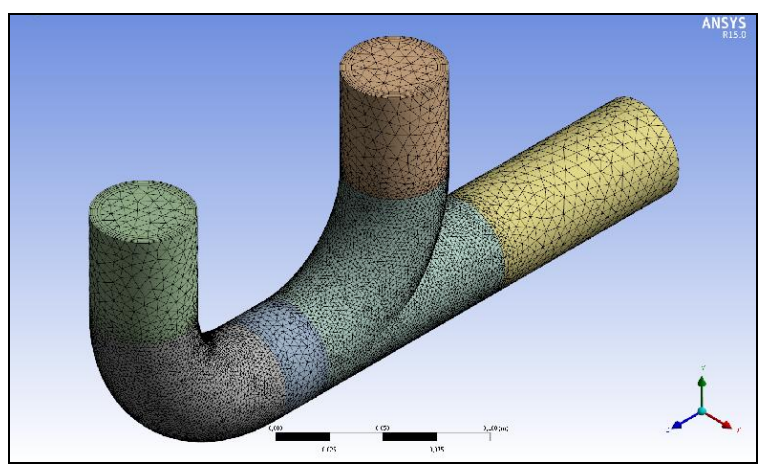

Fig. 5. Description of mesh on ram pump bodies for U-shape model

Table 1. Experiment Data of Ram Pump Bodies.

\begin{tabular}{|c|c|c|c|c|c|}
\hline $\begin{array}{l}\text { Ram } \\
\text { Pump } \\
\text { Body } \\
\text { Types }\end{array}$ & $\begin{array}{l}\text { Head } \\
\text { Input }\end{array}$ & $\begin{array}{l}\text { Head } \\
\text { Output }\end{array}$ & $\begin{array}{l}\text { Length } \\
\text { of } \\
\text { Impulse } \\
\text { Valve } \\
\text { (S) }\end{array}$ & $\begin{array}{c}\text { Static } \\
\text { Pressure } \\
\text { (Ps) }\end{array}$ & $\begin{array}{c}\text { Dynamic } \\
\text { Pressure } \\
\text { (Pd) }\end{array}$ \\
\hline & $m$ & $m$ & $m$ & Bar & Bar \\
\hline 1 & \multirow{3}{*}{1.7} & \multirow{3}{*}{4.3} & \multirow{3}{*}{0.02} & \multirow{3}{*}{0.2} & 1.2 \\
\hline 2 & & & & & 1.0 \\
\hline 3 & & & & & 1.4 \\
\hline
\end{tabular}

\section{Result and Discussion}

Based on the result of simulation, flow characteristic of ram pump can be seen in vector of pressure in Figure 6, Figure 7, Figure 8. Vector of pressure purposes to explain direction of flow that be occurred in different model of ram pump bodies. In Figure. 6, decreasing of pressure occurred in behind of impulse valve area where appeared wake phenomenon that indicated turbulent flow. Meanwhile, reduction of pressure was showed in $\mathrm{T}$-shape model on ram pump bodies where appeared any significantly wake in left side of impulse valve and right side of impulse valve that can be showed on Figure 7. On the other hand, wake phenomenon was produced by Y-shape model on left side of impulse valve; thus, flow direction was toward inlet 2 regions [4].

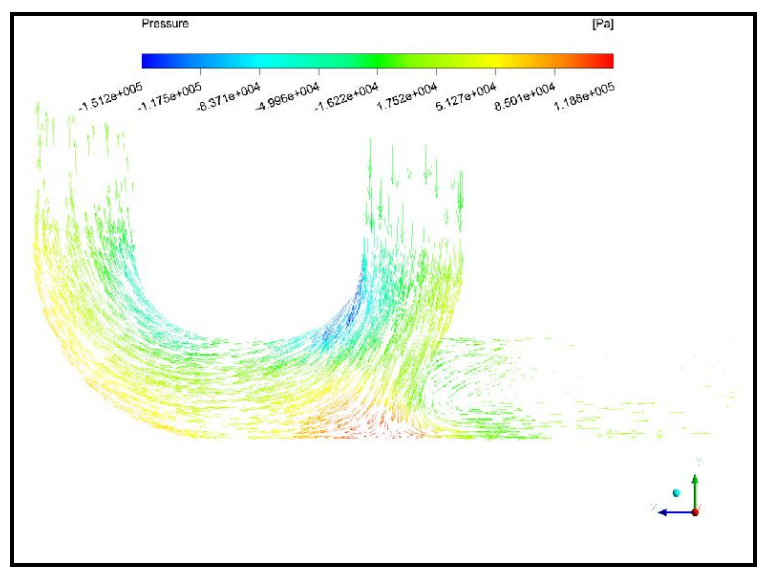

Fig. 6. Vector of pressure distribution of U-shape model on ram pump bodies with dynamic pressure of 1.2 bars. 
Corresponding with momentum theory, when a fluid flow through ram pump bodies is deflected depend on inner wall of pipe, there will be flow separation so it gives effect of dissipation energy in the form of turbulent. In Figure 9, Figure 10, Figure 11 showed characteristic scale that be expressed as a percent. Turbulent intensity is defined as root square of the velocity fluctuations and mean velocity (Reynolds averaged). The highest scale of turbulent intensity occurred in Y-shape model on ram pump bodies [3].

The turbulent kinetic energy is the mean kinetic energy per unit mass related with eddies in turbulent flow that indicates the strength of turbulent. Distribution of turbulent kinetic was showed in Figure 12, Figure 13, Figure 14 through pathlines contour. In area wake region, the highest turbulent kinetic energy located due to any increasing of velocity magnitude; thus, it was resistance of fluid flow toward outlet region. Comparing Figure 10, Figure 11, and Figure 12, it showed that Yshape model on ram pump bodies has highest value of turbulent kinetic than other models [4].

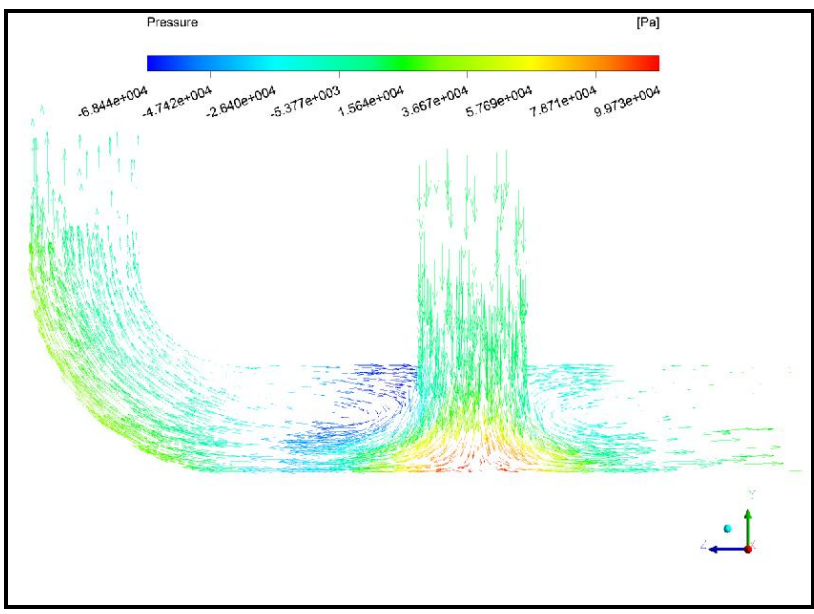

Fig. 7. Vector of pressure distribution of T-shape model on ram pump bodies with dynamic pressure of 1 bars.

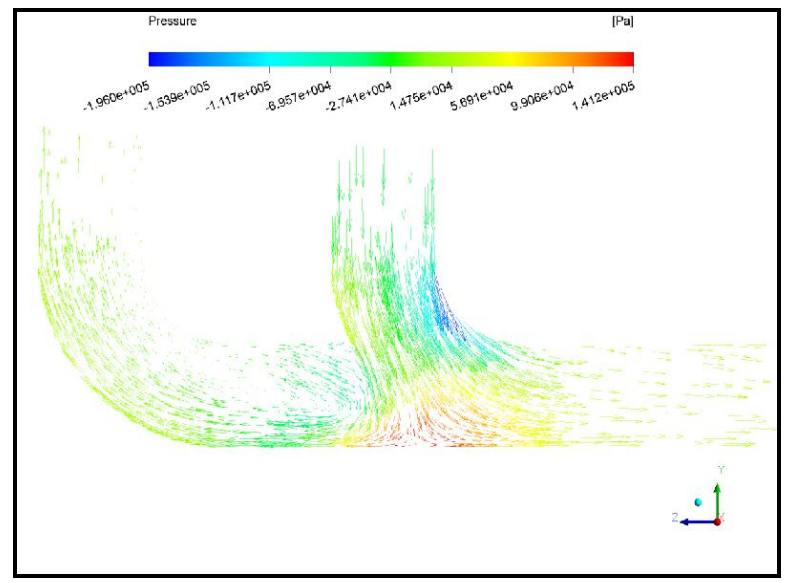

Fig. 8. Vector of pressure distribution of Y-shape model on ram pump bodies with dynamic pressure of 1.4 bars.

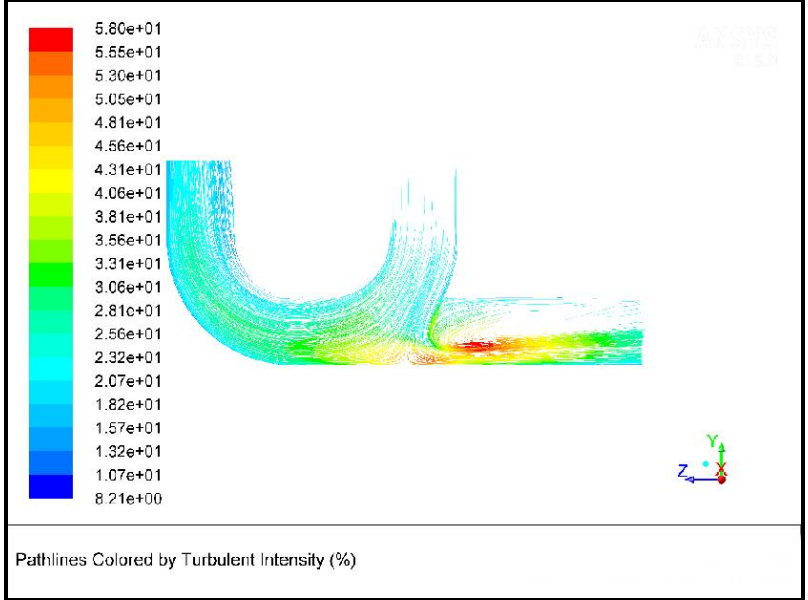

Fig. 9. Pathlines of turbulent intensity of U-shape model on ram pump bodies with dynamic pressure of 1.2 bars.

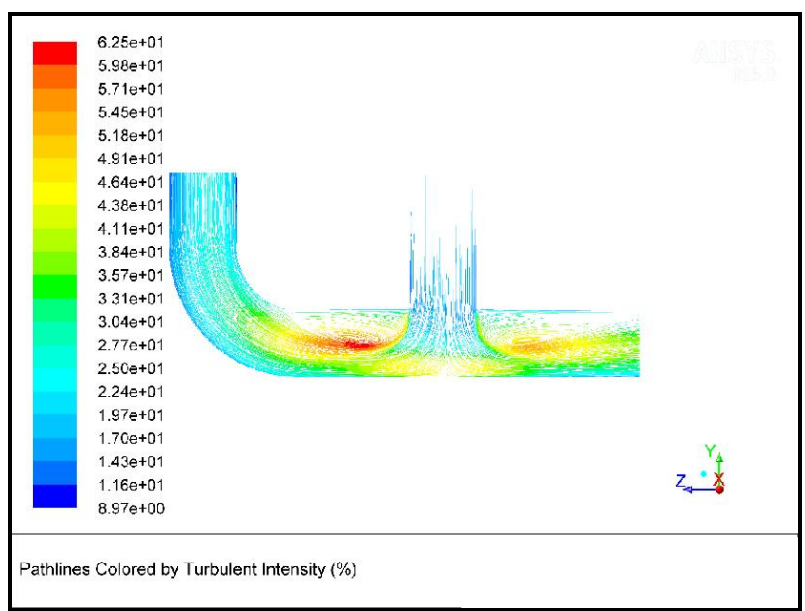

Fig. 10. Pathlines of turbulent intensity of T-shape model on ram pump bodies with dynamic pressure of 1 bars.

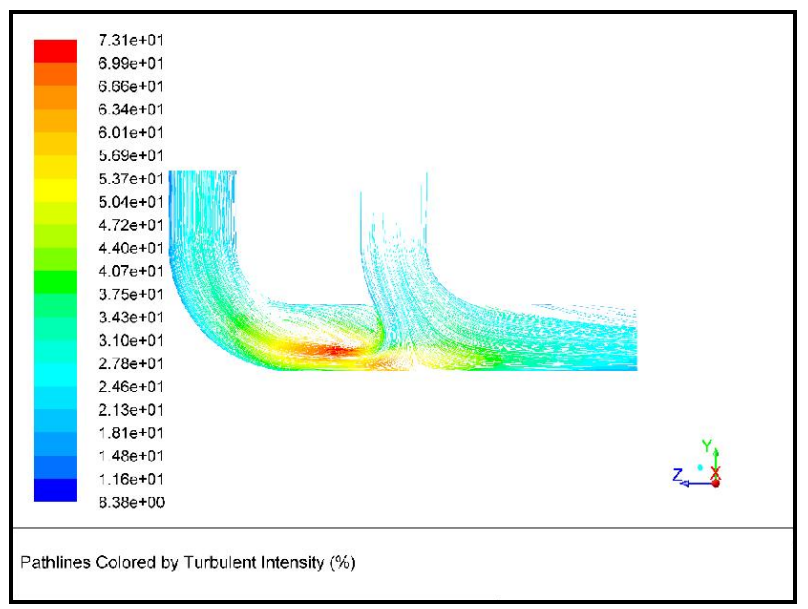

Fig. 11. Pathlines of turbulent intensity of Y-shape model on ram pump bodies with dynamic pressure of 1.4 bars. 


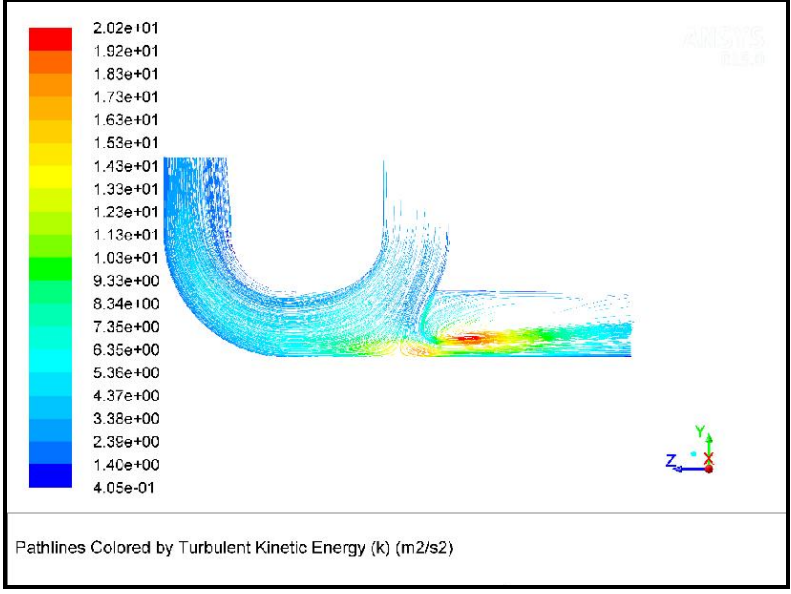

Fig. 12. Pathlines of turbulent kinetic energy of U-shape model on ram pump bodies with dynamic pressure of 1.2 bars.

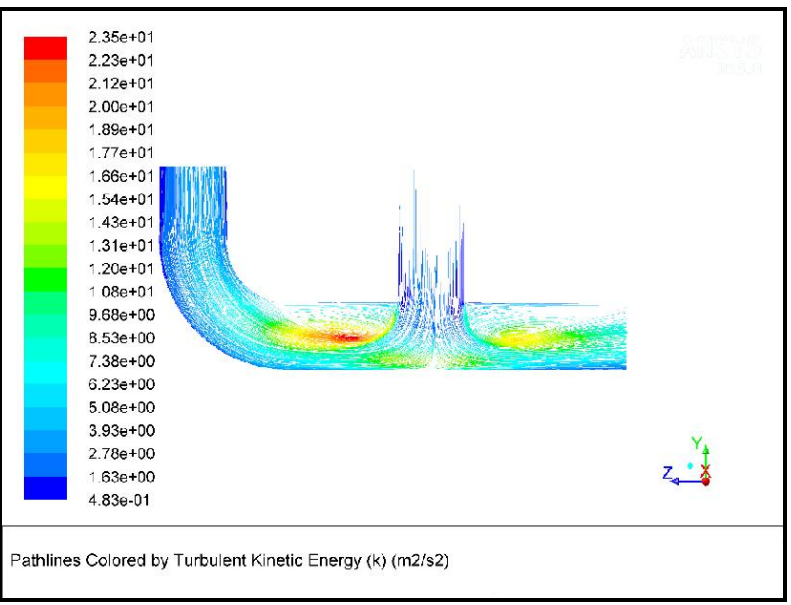

Fig 13. Pathlines of turbulent kinetic energy of T-shape model on ram pump bodies with dynamic pressure of 1 bars.

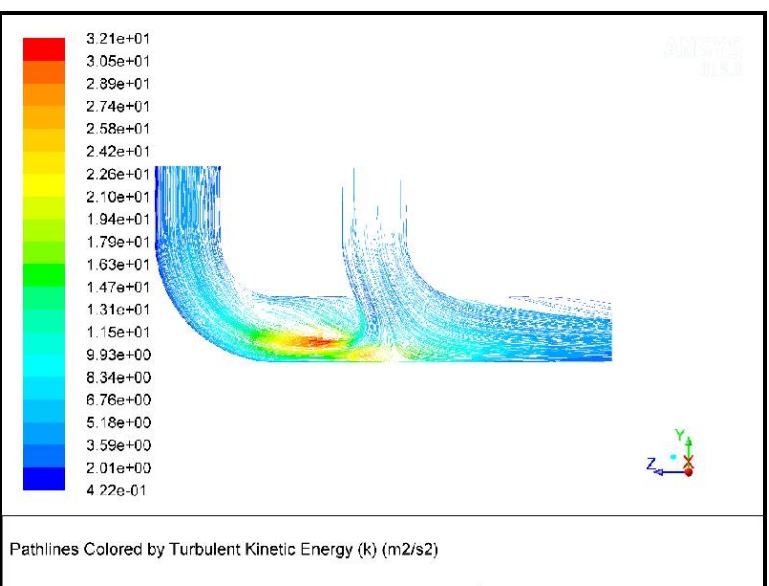

Fig 14. Pathlines of turbulent kinetic energy of Y-shape model on ram pump bodies with dynamic pressure of 1.4 bars.

\section{Conclusions}

In this study, a three-dimensional simulation was performed to see the flow characteristic on variation of ram pump bodies such as U-shape, T-shape, and Y-shape models through several parameters of fluid flow. The main conclusions based on the result of simulation to know the characteristic of fluid flow were summarized as follows:

1. Decreasing of pressure close to impulse valve either left side or right side showed that any wake phenomenon due to effect of dissipation energy; thus, it can be resistance of fluid flow toward outlet region.

2. The highest scale of turbulent intensity and turbulent kinetic were occurred in Y-shape model on ram pump bodies because effect of momentum that appeared in ram pump bodies.

\section{References}

1. T.D. Jeffery, T.H. Thomas, A.V. Smith. A guide to ram pump water supply systems. 3. Warwick University (2005).

2. Matthias Inthachot, Suchard Saehaeng, Agri. Sci. Procedia 5, 2 (2015).

3. J F Bingham, G P Blair, Proc. In. Mech. Eng. 199, $71(2001)$

4. Jing Shi, Mustapha Gourma. Pet. Sci. Eng. 151, 382 (2017) 